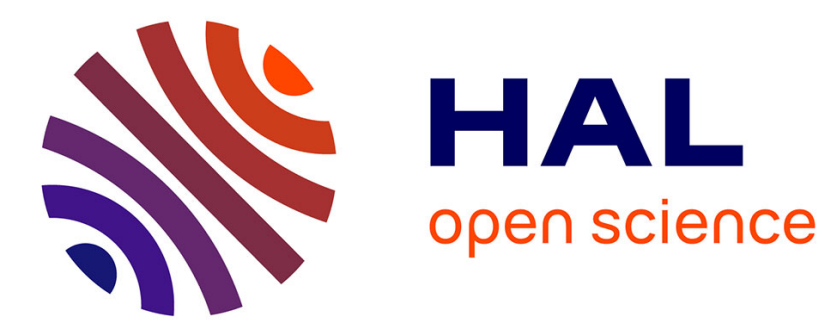

\title{
CONSTRUCTION OF LARGE PACKET RADIO NETWORKS
}

Jean-Claude Bermond, Pavol Hell, Jean-Jacques Quisquater

\section{To cite this version:}

Jean-Claude Bermond, Pavol Hell, Jean-Jacques Quisquater. CONSTRUCTION OF LARGE PACKET RADIO NETWORKS. Parallel Processing Letters, 1992, 02 (01), pp.3-12. 10.1142/S012962649200012X . hal-03200906

\section{HAL Id: hal-03200906 https://hal.science/hal-03200906}

Submitted on 16 Apr 2021

HAL is a multi-disciplinary open access archive for the deposit and dissemination of scientific research documents, whether they are published or not. The documents may come from teaching and research institutions in France or abroad, or from public or private research centers.
L'archive ouverte pluridisciplinaire HAL, est destinée au dépôt et à la diffusion de documents scientifiques de niveau recherche, publiés ou non, émanant des établissements d'enseignement et de recherche français ou étrangers, des laboratoires publics ou privés. 


\title{
CONSTRUCTION OF LARGE PACKET RADIO NETWORKS*
}

\author{
JEAN-CLAUDE BERMOND \\ CNRS, Université de Nice - Sophia Antipolis, 250 avenue A. Einstein, \\ Bat. 4, Sophia-Antipolis 1, 06560 Valbonne Cedex, France \\ PAVOL HELL \\ School of Computing Science, Simon Fraser University, Burnaby, \\ British Columbia, V5A IS6, Canada \\ JEAN-JACQUES QUISQUATER \\ Philips Research Laboratory, Louvain-la-Neuve, Belgium
}

\begin{abstract}
We outline constructions of packet radio networks (with time division multiplexing) that achieve much better parameters than those previously proposed. Given the desired diameter and number of slots per time frame, our networks seek to maximize the possible number of users. We model this as a problem of constructing large graphs or digraphs with given diameter and chromatic index, and relate it to extant work on large graphs with given diameter and maximum degree.
\end{abstract}

Keywords: Packet radio networks, interconnection networks, telecommunication networks, chromatic index, arc-chromatic index, maximum degree, diameter.

\section{Introduction}

In a recent article, Prohazka [17] considered the problem of designing packet radio networks which use time division multiplexing and have a diameter constraint. In particular, he investigated the maximum possible number of users of such a network, with diameter $D$ and with $f$ time slots per frame. Since a user cannot transmit and/or receive more than one packet at a time, this amounts to assigning time slots to channels (one channel for each ordered pair of communicating users) in such a way that the channels involving a particular user in either transmission or reception are all assigned different time slots. (Frequency division multiplexing was also used to prevent interference between users, but the number of bands was assumed sufficiently large to assign different frequency bands to all channels operating in the same time slot). Other work on packet radio networks may be found in [21].

\footnotetext{
*This research was supported by grants from $C^{3}$, NSERC and ASI.
} 
The above problem can be modeled by directed graphs: the users correspond to vertices, the channels to arcs, and assigning time slots corresponds to coloring the arcs so that incident arcs have different colors. Thus the problem is to determine the maximum number of vertices of a digraph with diameter $D$ whose arcs can be colored with $f$ colors.

Prohazka offered some general constructions of such digraphs. The number of vertices of his digraphs grows asymptotically as, roughly, $(f / 2)^{D / 2}$. He also compiled a table of such digraphs with values of $D$ up to ten and $f$ up to twenty. We shall obtain digraphs with $(f / 2)^{D}+(f / 2)^{D-1}$ vertices, and substantially improve many values listed in [17].

Prohazka's constructions all have an additional property. Namely, the digraphs constructed are all symmetric, i.e. all channels are bidirectional. This can be stated as a separate problem: Find the maximum number of vertices of a graph with diameter $D$ whose edges can be colored with $q$ colors in such a way that any two adjacent edges have different colors.

Our purpose in this paper is to point out the similarity of these problems with those of constructing large digraphs with diameter $D$ and maximum in- and outdegree $d$, and large graphs with diameter $D$ and maximum degree $\Delta$. Since there is an extensive literature on these subjects, we can exploit this similarity to derive our improvements of the results of [17].

The packet radio network is modeled by a digraph $G=(V, E)$ in which the vertices (elements of the set $V$ ) represent the users, and there is an arc (or directed edge, i.e. an element of the set $E$ ) $x y$ from vertex $x$ to vertex $y$. to indicate that user $x$ can transmit to user $y$. Thus the arcs represent unidirectional channels; a bidirectional channel joining $x$ and $y$ may be represented by two arcs $x y$ and $y x$. If all channels are bidirectional, the resulting digraph is symmetric, i.e. it has with each arc $x y$ also the arc $y x$. In this case we may consider instead an undirected graph with an edge $\{x, y\}$ replacing the two arcs $x y$ and $y x$.

We are only interested in digraphs which are strongly connected, i.e. digraphs in which there exists a directed path from any vertex $x$ to any vertex $y$. The length (number of arcs) of a shortest directed path from $x$ to $y$ is called the distance from $x$ to $y$ and denoted by $d(x, y)$. The diameter of a digraph $G$ is the maximum distance $d(x, y)$ over all vertices $x$ and $y$ of $G$. The outdegree of a vertex $x$ in the digraph $G$ is the number of arcs $x y$ in $G$, and is denoted by $d^{+}(x)$. The indegree $d^{-}(x)$ is defined analogously. Thus $d^{+}(x)$ is the number of channels $x$ can transmit to and $d^{-}(x)$ the number of channels $x$ can receive from. An arc-coloring of a digraph $G$ is a mapping assigning colors (labels) to the $\operatorname{arcs}$ of $G$ in such a way that two arcs having a common vertex obtain different colors. The arc-chromatic index of $G$ is the minimum number of colors which make an arc-coloring of $G$ possible.

An $(f, D)_{C}$-digraph is a digraph with diameter at most $D$ and arc-chromatic index at most $f$. We denote, by $n_{C}(f, D)$, the maximum number of vertices of an $(f, D)_{C}$-digraph. The problem mentioned in the introduction is to evaluate $n_{C}(f, D)$. 
All of the networks constructed in [17] are bidirectional, i.e. correspond to symmetric digraphs. The construction of symmetric $(f, D)_{C}$-digraphs may be considered as a separate problem. It is somewhat more convenient to discuss this problem in the context of undirected graphs, as explained above. All the above definitions have obvious analogues for undirected graphs: For a connected graph $G$, the distance $d(x, y)$ is the length of a shortest path from $x$ to $y$, and the diameter of $G$ is the greatest $d(x, y)$ for all pairs of vertices $x, y$ of $G$. The maximum degree of $G$ is the largest number of edges incident with any vertex of $G$, and the edge-chromatic index of $G$ is the minimum number of colors which can be assigned to the edges of $G$ so that two edges having a common vertex obtain different colors.

A $(q, D)_{C}$-graph is a graph with diameter at most $D$ and edge-chromatic index at most $q$. We denote by $n_{C}^{*}(q, D)$ the maximum number of vertices in a $(q, D)_{C^{-}}$ graph.

A remark about our notation: The function $n_{C}$ refers to the number of vertices in largest directed graph with given chromatic index and diameter. For the undirected version of the same problem we add the superscript $*$. We shall have other versions of these functions later in the paper. In particular, omitting the subscript $C$ signifies that chromatic index is replaced by maximum degree, e.g. $n$ is the function corresponding to largest digraphs with given maximum in- and out- degree and diameter, cf. below. Adding a superscript $*$ concerns again the corresponding undirected version of the problem. Adding other superscripts $(s, b, t)$ will refer to other restricted versions of the problem.

\section{Graphs}

A $(\Delta, D)$-graph is a graph with diameter at most $D$ and maximum degree at most $\Delta$. We denote by $n^{*}(\Delta, D)$ the maximum number of vertices in a $(\Delta, D)$ graph. We begin by exploring the relationship between $(\Delta, D)$-graphs and $(q, D)_{C^{-}}$ graphs. Indeed, the construction of large $(\Delta, D)$-graphs and the evaluation of $n^{*}(\Delta, D)$ is a challenging problem that has been widely considered in recent years, see, for example, the survey [3], or the special issue [22]. It is clear that in any edge-coloring of a graph $G$, a vertex of degree $\Delta$ needs at least $\Delta$ colors for its incident edges. Thus the edge-chromatic index $q$ of $G$ is at least the maximum degree $\Delta$. On the other hand, a theorem of Vizing (see [9], Chapter 6), ensures that the edge-chromatic index $q$ is at most $\Delta+1$ (in other words, any graph $G$ with maximum degree $q-1$ admits an edge-coloring with $q$ colors). Thus we have the following observations.

Proposition 1. $n^{*}(q-1, D) \leq n_{C}^{*}(q, D) \leq n^{*}(q, D)$.

Proposition 1 is useful, on the one hand, to obtain an upper bound on $n_{C}^{*}(q, D)$ by using the upper bound on $n^{*}(\Delta, D)$ known as the Moore bound [7]. The Moore bound is obtained by noting that there are, in a $(\Delta, D)$-graph, at most $\Delta(\Delta-1)^{k-1}$ vertices at distance $k$ from any fixed vertex. Thus,

$$
n^{*}(\Delta, D) \leq 1+\Delta+\Delta(\Delta-1)+\ldots+\Delta(\Delta-1)^{D-1} \text {. }
$$


We conclude that

$$
n_{C}^{*}(q, D) \leq 1+q+q(q-1)+\ldots+q(q-1)^{D-1} .
$$

On the other hand, we also use Proposition 1 by appealing to any known construction of large $(q-1, D)$-graphs to obtain a lower bound on $n_{C}^{*}(q, D)$. This improves practically all the lower bounds of [17]. For instance, the value $n_{C}^{*}(10,10)$, bounded below by 206660 in [17], is at least $n^{*}(9,10)$ according to Proposition 1 . Thus we may use a construction due to Campbell [10], showing this value to be bounded by 19845936 .

Further improvement will occur with many constructions of large $(\Delta, D)$-graphs in which we can show an edge-coloring with $\Delta$ (rather than $\Delta+1$ ) colors. An example of a $(\Delta, D)$-graph that is colorable with $\Delta$ colors appears in Fig. 1 , where a largest possible $(3,3)$-graph on 20 vertices is edge-colored with 3 colors (cf. [3]). Thus $n_{C}^{*}(3,3)=n^{*}(3,3)=20$ (to be compared with the bound of 14 in [17]; Fig. 4).

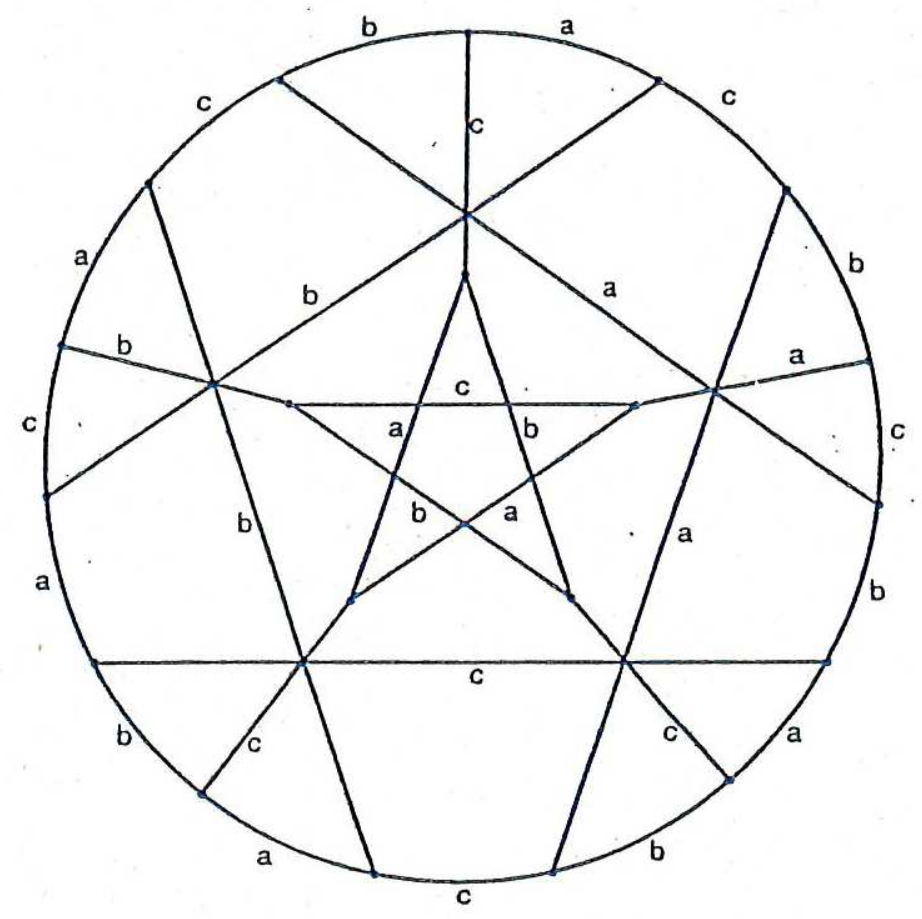

Fig. 1. A largest $(3,3)_{C}$-graph, with a 3-coloring of its edges.

A $\Delta$-coloring always exists for a bipartite $(\Delta, D)$-graph. It is well known that the maximum degree and the edge-chromatic index of a bipartite graph are equal [9]. Thus $n_{C}^{*}(q, D)$ is at least as large as the number of vertices of any bipartite $(q, D)$ graph. This is often useful to know as many well known network constructions are bipartite - such as the hypercube, or the star graph [1]. Taking large bipartite $(q, D)$-graphs from [11] or [12], we further improve the lower bounds on many $n_{C}^{*}(q, D)$; for instance, we obtain $n_{C}^{*}(10,10) \geq 47059200$.

It is also shown in [16] that there exist bipartite $(q, D)$-graphs with $2(q / 2)^{D-1}+$ $2(q / 2)^{D-3}$ vertices. Thus we can conclude that $n_{C}^{*}(q, D) \geq 2(q / 2)^{D-1}+2(q / 2)^{D-3}$. 
This is an improvement over the asymptotic bounds discussed in Sec. IV of [17], which are roughly of the order $(q / 2)^{D / 2}$ as $q$ tends to infinity. (Cf. also [11] for a slightly smaller improvement.)

It has been our experience that in most examples of the largest known $(\Delta, D)$ graphs, an edge-coloring with $\Delta$ colors is possible. This is the case in particular for the undirected de Bruijn and Kautz graphs. The latter are $(2 d, D)$-graphs with $d^{D}+d^{D-1}$ vertices, obtained by ignoring the arc-directions of the Kautz digraphs $K(d, D)$ defined in the next section. In particular, we conclude (cf. Corollary 5 ):

Corollary 2. $(q / 2)^{D}+(q / 2)^{D-1} \leq n_{C}^{*}(q, D) \leq q(q-1)^{D-1}+q(q-1)^{D-2}+\ldots$ $+q+1$.

The lower bound in the above corollary is a substantial improvement of the constructions in [17]. Even though it is quite far from the theoretical upper bound (derived from the Moore bound), we are confident that, in full generality, further improvements will not be easy. The reason for this is that any improved constructions would in particular provide better bounds for the much studied parameter $n^{*}(\Delta, D)$, via Proposition 1. This does not mean, of course, that particular values of $n_{C}^{*}(q, D)$, or even infinite sequences of such values, could not be better estimated.

In all the cases below, the best known $(\Delta, D)$-graphs have been shown colorable with $\Delta$ colors:

- the $(2 k+1,2 m)$-graphs $(k \geq 2)$ with $k^{m}(k+1)^{m}$ vertices, known as the sequence graphs (cf. [13], [15]).

- the $(p+1,2)$-graphs with $p^{2}+p+1$ vertices, $p$ a prime power, arising from the projective planes (cf. [3], [19]).

- some of the best known $(3, D)$-graphs, in particular those with $D=4$ and 38 vertices, $D=5$ and 70 vertices [15], $D=16$ and 128 vertices, $D=7$ and 184 vertices, and $D=8$ and 320 vertices [18].

- the $(3, D)$-graphs, for $D=2^{k}-2$, with $2 \cdot 2^{D}$ vertices obtained by substituting an edge in each vertex of the de Bruijn graph $B(2, D)$, cf. below (and similar $(\Delta, D)$-graphs when $\Delta$ is one plus a power of two) [6].

We conclude this section by noting that it is not always the case that the best $(\Delta, D)$-graphs are $\Delta$-edge-colorable. The unique largest $(3,2)$-graph is the (tenvertex) Petersen graph, cf. [9]; it is known that it is not 3-edge-colorable. There is no graph on 9 vertices with all degrees equal to 3 , and if a nine-vertex graph has a vertex of degree two or less, then counting like in the Moore bound, starting from this vertex, we see that it cannot have diameter 2 , and hence $n^{*}(3,2)$ is at most eight. In fact, there is a $(3,2)_{C}$-graph with eight vertices, obtained from the cycle $0,1, \ldots, 7,0$ by adding the edges $\{0,4\},\{1,5\},\{2,6\},\{3,7\}$. Therefore $n_{C}^{*}(3,2)=8$.

\section{Digraphs}

Let us now consider the construction of $(f, D)_{C}$-digraphs, i.e. digraphs with diameter at most $D$ and arc-chromatic index at most $f$. 
Since graphs correspond to symmetric digraphs, we have already constructed large $(f, D)_{C}$-digraphs. Let $n_{C}^{s}(f, D)$ denote the maximum number of vertices in a symmetric $(f, D)_{C^{-}}$-digraph. Any $(q, D)_{C}$-graph yields a symmetric $(2 q, D)_{C^{-}}$ digraph by replacing each edge $\{x, y\}$ by the arcs $x y$ and $y x$. Moreover, an edgecoloring of the graph gives rise to an arc-coloring of the digraph with twice as many colors. Hence $n_{C}(2 f, D) \geq n_{C}^{s}(2 f, D)=n_{C}^{*}(f, D)$.

Moreover, we expect to be able to construct even larger $(f, D)_{C}$-digraphs when the symmetry condition is removed. Note that we know from Corollary 2, that, roughly, $n_{C}^{s}(2 f, D) \leq f^{D}$. It is our objective to construct (non-symmetric) $(f, D)_{C^{-}}$ digraphs larger than this upper bound for symmetric digraphs.

Consider first another special class of $(f, D)_{C}$-digraphs. We shall say that an $(f, D)_{C}$-digraph is balanced if both $d^{+}(x) \leq f / 2$ and $d^{-}(x) \leq f / 2$ for every vertex $x$. Let $n_{C}^{b}(f, D)$ be the maximum number of vertices in a balanced $(f, D)_{C}$-digraph. A $(d, D)$-digraph is a digraph $G$ with diameter at most $D$ and maximum in- and out- degree at most $d$. Note that while this notion is analogous with the notion of a $(\Delta, D)$-graph, we do not have here the close analogy we had between $(\Delta, D)$ graphs and $(\Delta, D)_{C}$-graphs. Specifically, the reader should be aware that a $(2 d, D)$ digraph has maximum in- and out- degree $2 d$, while a $(2 d, D)_{C}$-digraph has, in particular, each vertex with $d^{+}+d^{-}$bounded by $2 d$. Thus, in some sense; a $(d, D)$ digraph is automatically balanced. We write $n(d, D)$ for the maximum number of vertices of a $(d, D)$-digraph. (If we were to define the function $n^{b}(d, D)$ as the maximum number of vertices in a balanced $(d, D)$-digraph, we would have $\left.n(d, D)=n^{b}(d, D)\right)$. We have the following very close relationship of $n_{C}^{b}(f, D)$ and $n(d, D)$.

Proposition 3. $n(f-1, D) \leq n_{C}^{b}(2 f, D) \leq n(f, D)$.

Proof. The first inequality follows, as before, from a version of Vizing's theorem [9]. In fact, any $(f-1, D)$-digraph with edge-directions ignored is an undirected multigraph with edge multiplicities at most two. The maximum degree in this multigraph is at most $2 f-2$ and Vizing's theorem implies that the edges of the multigraph, and hence also the arcs of the digraph, can be colored with $2 f$ colors. Thus any $(f-1, D)$-digraph is also a balanced $(2 f, D)_{C}$-digraph. The second inequality is trivial. It simply says that in a balanced $(2 f, D)_{C}$-digraph both inand out- degrees are at most $f$.

In view of Proposition 3, we can again use the known constructions of large $(d, D)$-digraphs. The best general construction among these is the following (we assume $d \geq 2)$ : The de Bruijn digraph $B(d, D)$ has as its vertices all strings of length $D$ over the alphabet $\{0,1, \ldots, d-1\}$; there is an arc from a vertex $a_{1} a_{2} a_{3} \ldots a_{D}$ to all vertices $a_{2} \dot{a}_{3} \ldots a_{D} a$ with $a$ in $\{0,1, \ldots, d-1\}$. The $K a u t z$ digraph $K(d, D)$ has as its vertices all those strings of length $D$ over the alphabet $\{0,1, \ldots, d\}$ in which consecutive characters are distinct; there is an arc from a vertex $a_{1} a_{2} a_{3} \ldots a_{D}$ to all vertices $a_{2} a_{3} \ldots a_{D} a$ with $a$ from $\{0,1, \ldots, d\}$ and distinct from $a_{D}$. It is easy to see (cf. [4], [14], 20]) that both $B(d, D)$ and $K(d, D)$ are $(d, D)$-digraphs. Moreover, 
the digraph $B(d, D)$ has $d^{D}$ vertices and the digraph $K(d, D)$ has $d^{D}+d^{D-1}$ vertices. We immediately obtain, via the proof of Proposition 3, large (balanced) $(2 d+2, D)_{C}$-digraphs. However, we were able to prove (see [5]) that $B(d, D)$ and $K(d, D)$ are in fact $(2 d, D)_{C}$-digraphs:

Proposition 4. Both $B(d, D)$ and $K(d, D)$ (for $D \geq 2$ ) have arc-chromatic index $2 d$.

From this we find a good estimation of the maximum number of vertices in a balanced $(f, D)_{C}$-digraph: Consider the Moore bound for $n(d, D)$, obtained by noting that in a $(d, D)$-digraph there are at most $d^{k}$ vertices of distance $k$ from a fixed vertex.

Corollary 5. $f^{D}+f^{D-1} \leq n_{C}^{b}(2 f, D) \leq f^{D}+f^{D-1}+\ldots+1$.

It follows, e.g. that the Kautz digraph $K(3,3)$ on 36 vertices (cf. Fig. 2) yields $n_{C}(6,3) \geq 36$. This improves on the value 14 obtained by Prohazka and on the value 20 obtained for $n_{C}^{*}(3,3)$ in the preceding section.

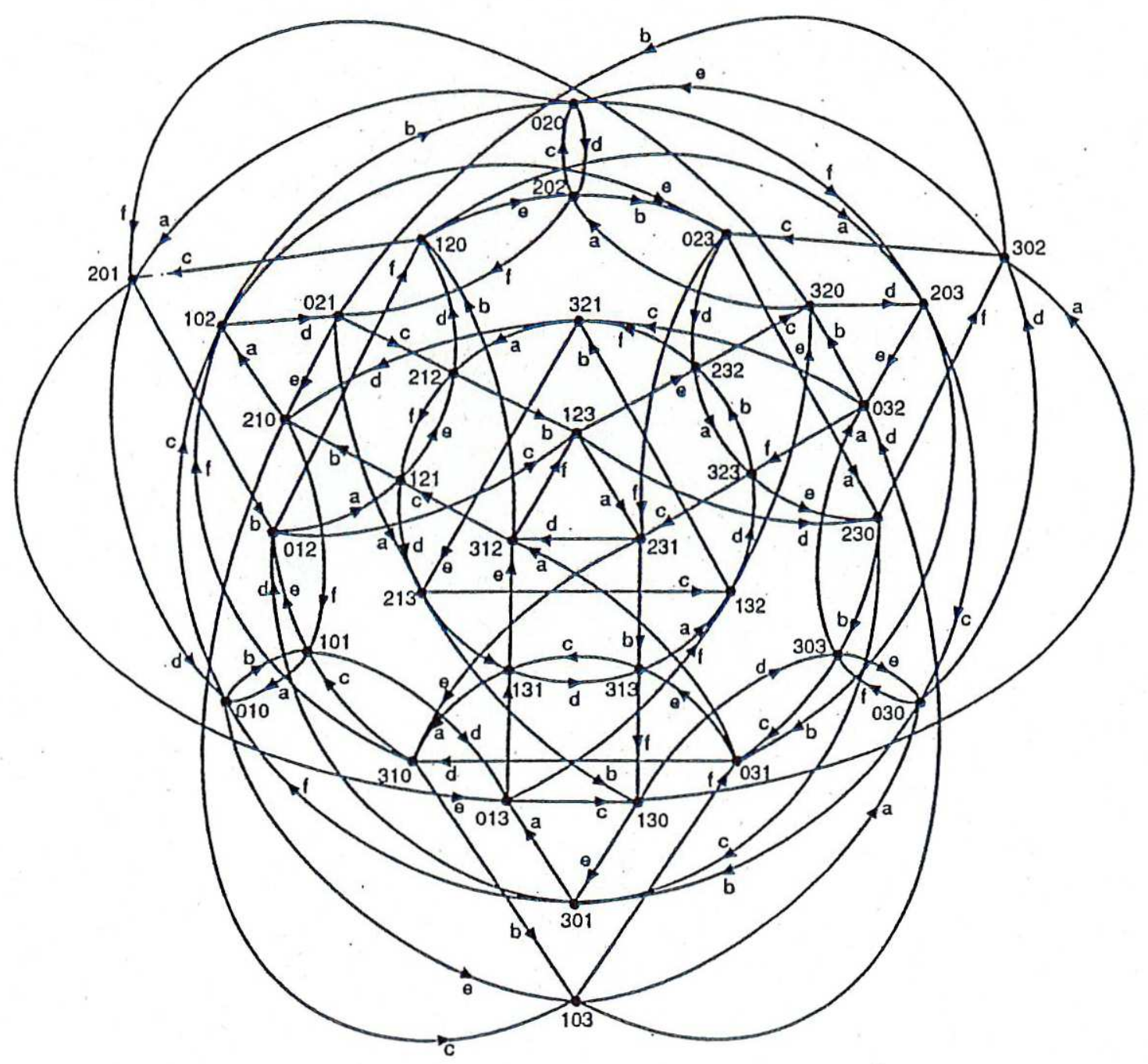

Fig. 2. The $(6,3)_{C}$-digraph $K(3,3)$, with a 6 -coloring of the arcs. 
Proposition 4 is a special case of a more general result on line digraphs [5]. Using this general result and the family constructed in [14], J. Bond [8] has recently obtained a slight improvement of Corollary 5 in the case of $2 d=4$. (As always, this was done by finding a 4 -arc-coloring of a large $(2, D)$-digraph). In fact he has shown that if $D \geq 6$, then $n_{C}(4, D) \geq 25.2^{D-4}$, rather than $\geq 24.2^{D-4}$ implied by Corollary 5 .

We now return to the general problem of large (not necessarily balanced) $(f, D)_{C}$-digraphs. This problem also has an analogous problem concerned with the degree; however, that problem does not seem to have been previously studied. Let $n^{t}(d, D)$ denote the maximum number of vertices of a digraph of diameter $D$ such that the total degree $d^{+}(x)+d^{-}(x)$ of each vertex $x$ is at most $d$.

Note that any $(d, D)$-digraph has maximum total degree bounded by $2 d$. Moreover, as the digraph is strongly connected, if $d^{+}(x)+d^{-}(x) \leq 2 d$ then $1 \leq d^{+}(x) \leq$ $2 d-1$ and $1 \leq d^{-}(x) \leq 2 d-1$. Therefore

$$
n(d, D) \leq n^{t}(2 d, D) \leq n(2 d-1, D) .
$$

The close relationship between $n^{t}(d, D)$ and $n_{C}(f, D)$ is made explicit in the following proposition, proved along the lines of the proof of Proposition 3.

Proposition 6. $n^{t}(f-2, D) \leq n_{C}(f, D) \leq n^{t}(f, D)$.

One can argue in a spirit similar to proving the Moore bound, that $n^{t}(2 f, 2) \leq$ $f^{2}+f$. Thus combining Propositions 3 and 6 , and Corollary 5 we obtain the precise value of the parameter $n_{C}(2 f, 2)$.

Corollary 7. $n_{C}(2 f, 2)=f^{2}+f$.

\section{Conclusions}

We described several constructions (and referred to many others) of large graphs with given diameter and maximum degree $\Delta$ which can be edge-colored with $\Delta$ colors. As a consequence we obtained the following lower bounds on $n_{C}^{*}(\Delta, D)$ :

- $n_{C}^{*}(3,2)=8$

- $n_{C}^{*}(3,3)=20, n_{C}^{*}(3,4) \geq 32, n_{C}^{*}(3,5) \geq 56, n_{C}^{*}(3,6) \geq 128, n_{C}^{*}(3,7) \geq 184$ and $n_{C}^{*}(3,8) \geq 320$

- $n_{C}^{*}(10,10) \geq 47059200$

- $n_{C}^{*}\left(3,2^{k}-2\right) \geq 2^{2^{k}-1}$

- $n_{C}^{*}(2 f, D) \geq f^{D}+f^{D-1}$

- $n_{C}^{*}(2 f+1,2 m) \geq f^{m}(f+1)^{m}$

- $n_{C}^{*}(p+1,2) \geq p^{2}+p+1$.

For the corresponding problem on digraphs we have $n_{C}(2 f, D) \geq n_{C}^{*}(f, D)$, and in addition:

- $n_{C}(6,3) \geq 36$

- $n_{C}(2 f, D) \geq f^{D}+f^{D-1}$ 
- $n_{C}(4, D) \geq 25.2^{D-4}$

- $n_{C}(2 f, 2)=f^{2}+f$.

From a practical perspective, we suggest the following technique to construct large $(q, D)_{C}$-graphs for given values of $q$ and $D$. Consider first the largest known $(q, D)$ graphs. If any such graph can be shown to admit an edge-coloring with $q$ colors, one should use it. Otherwise consider $(q, D)$-graphs for which a $q$-edge-coloring is known and which still have a relatively large number of vertices. In any case, we can use, for instance, the largest known bipartite $(q, D)$-graph. Similar comments apply in the case of $(f, D)_{C}$-digraphs.

Finally, we note that the best constructions we obtained for the case of general digraphs are better than the theoretical upper bounds for symmetric digraphs compare the lower bound in Corollary 5 with the rough upper bound $n_{C}^{s}(2 f, D) \leq$ $f^{D}$ mentioned at the beginning of Sec. 4. Thus there is a heavy penalty for requiring radio packet networks to be symmetric. On the other hand, it appears that requiring them to be balanced is not an obstacle, and in fact all of the best constructions happen to be balanced.

We thank J. Bond, M. A. Fiol, C. Delorme, and P. Solé for their interest in, and contributions to, this paper.

\section{References}

[1] S. B. Akers and B. Krishnamurthy, A group-theoretic model for symmetric interconnection networks, IEEE Trans. Comput. 38 (1989) 555-566.

[2] D. Ameter and Max Degree; Graphs and Interconnection Networks, in preparation.

[3] J.-C. Bermond, C. Delorme and J.-J. Quisquater, Strategies for interconnection networks: Some methods from graph theory, J. Parallel Distri. Comput. 3 (1986) 433-449.

[4] J.-C. Bermond and C. Peyrat, The de Bruijn and Kautz networks: a competition for the hypercube?, Hypercube and Distributed Computers, Proc. First European Colloquium on Hypercubes, Rennes, Oct. 1989 (Elsevier Science, 1989) 279-293.

[5] J.-C. Bermond and P. Hell, Even factorisations and the chromatic index of the Kautz and de Bruijn networks, submitted to J. Graph Theory.

[6] J.-C. Bermond and P. Hell, in preparation.

[7] N. Biggs, Algebraic Graph Theory, Cambridge Tracts in Math. 67 (Cambridge University Press, 1974).

[8] J. Bond, personàl communication.

[9] J. A. Bondy and U. S. R. Murty, Graph Theory with Applications (North Holland, 1976).

[10] L. Campbell, Dense group networks, to appear in DAMIN, the Special Issue of Discrete Applied Mathematics on Interconnection Networks.

[11] C. Delorme, Large bipartite graphs with given degree and diameter, J. Graph. Theory 9 (1985) 325-334.

[12] C. Delorme and J.-J. Quisquater, Some new compound graphs, to appear in DAMIN, the Special Issue of Discrete Applied Mathematics on Interconnection Networks.

[13] M. A. Fiol, J. L. A. Yebra and J. Fabrega, Sequence graphs and interconnection networks, Ars Combinatoria 16A (1983) 7-13.

[14] M. A. Fiol, J. L. A. Yebra and I. Alegre, Line digraph iterations and the $(d, k)$-digraph problem, IEEE Trans. Comput. 33 (1984) 400-403. 
[15] M. A. Fiol, personal communication.

[16] M. A. Fiol and J. L. A. Yebra, Dense bipartite subgraphs, J. Graph Theory, to appear.

[17] C. G. Prohazka, Bounding the maximum size of a packet radio network, IEEE Trans. Comput. 37 (1988) 1184-1190.

[18] J.-J. Quisquater, manuscript, 1990.

[19] P. Solé, personal communication, 1990.

[20] M. R. Samatham and D. K. Pradhan, The de Bruijn multiprocessor network: A versatile parallel processing and sorting network for VLSI, IEEE Trans. Comput. 38, 4 (1989) $567-581$.

[21] D. Towsley and C. G. Prohazka, Topology design for time slotted packet radio networks, 1981 IEEE Int. Symp. on Information Theory. Abstracts of Papers, Santa Monica, CA, USA, Feb. 1981 (IEEE, 1981) 121.

[22] DAMIN, Special Issue of Discrete Applied Mathematics on Interconnection Networks, Ed. J.-C. Bermond, to appear. 